BULL. AUSTRAL. MATH. SOC.

VOL. 9 (1973), 1-9.

\title{
A remark on a mean value theorem of Alexander Weinstein in Generalized Axially Symmetric Potential Theory
}

\section{J.B. Diaz and John G. Leschen}

\author{
Cordially dedicated to Professor Alexander Weinstein, \\ on the occasion of his Seventyseventh birthday, January $21,1974$.
}

This note contains the proof of an extension of Alexander Weinstein's mean value theorem for Generalized Axially Symetric Potential Theory.

\section{Introduction}

In his, now classical, paper [4], on Generalized Axially Symmetric Potential Theory (GASPT), Weinstein proved a mean value theorem, which he states as follows (see page 344 of [4]; the notation (WT) is introduced in the present note).

"MEAN VALUE THEOREM.

$$
\phi(0,0) \int_{0}^{\pi} \sin ^{p} \theta d \theta=\int_{0}^{\pi} \phi(x, y) \sin ^{p} \theta d \theta,
$$

where $\theta$ denotes the poiar angle. The integral on the right-hand side is taken over $a$ half-circle of arbitrary radius $a$.

Received 5 February 1973. 
A similar formula holds obviously for $\phi\left(x_{0}, 0\right)$ at any regular point $\left(x_{0}, 0\right)$ of $\phi . "$

In this quotation from [4], $\phi(x, y)$ is a solution of the partial differential equation (see page 343 of [4]):

$$
y\left(\phi_{x x}+\phi_{y y}\right)+p \phi_{y}=0,
$$

which is even in $y$, is analytic in the two real variables $(x, y)$ in an open set which intersects the $x$-axis, and is such that the partial derivative $\frac{\partial \phi}{\partial y}$ vanishes for $y=0$.

(The notations (WT) and (GP) have been introduced in the present note, for convenience of reference.)

\section{Weinstein's mean value theorem for $p>-1$}

Weinstein's development of his mean value theorem (WT) is based on the assumptions that $p>0$ and that $\phi$ is a solution of (GP) which is even in $y$ and is analytic in a region containing a segment of the $x$-axis. Weinstein also uses the fact that there is an associated "stream function" $\psi$ which satisfies the partial differential equation

$$
y\left(\psi_{x x}+\psi_{y y}\right)-p \psi_{y}=0
$$

However, it is clear that the definite integral on the right hand side of (WT) exists, for $p>-1$, whenever the integrand function $\phi(x, y)$ is continuous on $0 \leq \theta \leq \pi$. Furthermore, the premise that $\phi$ is an even function of $y$ appears to be somewhat unnecessary, specially when one considers that any solution of (GP) which is "regular" on a portion of the singular line $y=0$, must be such that, if $p \neq 0$, its partial derivative $\frac{\partial \phi}{\partial y}$ vanishes on the $x$-axis.

We are, therefore, led to ask whether Weinstein's mean value theorem (WT) might hold for $p>-1, p \neq 0$, with weakened hypotheses on the function $\phi$. An affirmative answer to this question is provided by the following extension of the Weinstein Theorem (WT):

THEOREM. Let $p>-1, p \neq 0$. Let $G$ be an open set in the upper half plane $y>0$, and suppose that the boundary of $G$ contains a non- 
degenerate open segment $I$ of the $x$-axis. In $G$, let $\phi(x, y) \in c^{2}$, with bounded second derivatives, be a real valued solution of (GP). In $G \cup I$, let $\phi \in C$. Let $x_{0}$ and $R, R>0$, be any real numbers such that the semicircle $\left(x-x_{0}\right)^{2}+y^{2} \leq R^{2}, y \geq 0$, lies entirely in $G \cup I$. Then, (WT) holds.

Proof. (It is true, as follows from a known general theorem, that any solution, of class $c^{2}$, of the elliptic partial differential equation (GP), must necessarily be analytic, in $(x, y)$, in $G$. However, this fact is not used in the present proof.)

Since (GP) is invariant under $x$-translations, the center of the semicircle may be taken to be the origin; thus, $x_{0}=0$. With $r$ and $\theta$ the usual polar coordinates, we define the "fan-shaped" region

$$
D(\alpha, \beta)=\{(r, \theta) \mid \alpha R<r<R, \beta<\theta<\pi-\beta\},
$$

where $0<\alpha<1$ and $0<\beta<\pi / 2$. Clearly, $D(\alpha, \beta)$ lies in $G$.

The proof will be based on the application of two Green's identities for the partial differential operator occurring in equation (GP), which are to be found in Weinstein [4, page 343, equations (4) and (5)]:

$$
\iint_{R} y^{p}\left(\phi_{x x}+\phi_{y y}+p y^{-1} \phi_{y}\right) d x d y=\int_{C} y^{p} \frac{\partial \phi}{\partial n} d x
$$

(5) $\iint_{R}\left[\phi^{*} \operatorname{div}\left(y^{p} \operatorname{grad} \phi\right)-\phi \operatorname{div}\left(y^{p} \operatorname{grad} \phi^{*}\right)\right] d x d y=\int_{C} y^{p}\left(\phi^{*} \frac{\partial \phi}{\partial n}-\phi \frac{\partial \phi^{*}}{\partial n}\right) d s$,

where $C$ is the boundary of a domain $R$, the letter $s$ denotes arc length on $C$, and $n$ is the exterior normal to $C$, and $\phi$ and $\phi^{*}$ are "regular" functions (of class $c^{2}$ in. G). Identities (4) and (5) were given by Weinstein for $p>0$, but they remain valid for any real $p$.

Several inequalities, which will be needed in the course of the proof, will now be established. Recall that, by hypothesis, the first and second partial derivatives of $\phi$ are bounded in $G$. Thus, there is a positive number $M$ that is a common upper bound of $\left|\phi_{x}\right|,\left|\phi_{y}\right|,\left|\phi_{x x}\right|,\left|\phi_{y y}\right|$, in $G$. Then, as a consequence of the mean value theorem of the differential calculus for functions of two variables, given $\alpha$ and $\theta$, there is a 
"mean value point" $Q$, interior to the straight line segment joining the origin $(0,0)$ to the point $(\alpha R, \theta)$, where $0<\alpha<1$ and $0<\theta<\pi$, such that (with $\phi_{0}=\phi(0,0)$ ):

$$
\phi(\alpha R, \theta)-\phi_{0}=(\alpha R \cos \theta) \cdot \phi_{x}(Q)+(\alpha R \sin \theta) \cdot \phi_{y}(Q) .
$$

Therefore,

$$
\left|\phi(\alpha R, \theta)-\phi_{0}\right| \leq 2 M \alpha R, \text { for } 0<\theta<\pi
$$

Since $\phi$ is continuous on $G \cup I$, the inequality just written continues to hold on the closed interval $0 \leq \theta \leq \pi$. Further, one has

$$
\lim _{\alpha \rightarrow+0} \phi(\alpha R, \theta)=\phi_{0}
$$

with the convergence being uniform on the closed interval $0 \leq \theta \leq \pi$. Also, it follows, directly from (GP), that

$$
\left|\phi_{y}\right|=\left|y\left(\phi_{x x}+\phi_{y y}\right) / p\right| \leq 2 y M /|p|
$$

in $G$.

Now, consider two points of the closure of $D(\alpha, \beta)$, with polar coordinates $(r, \beta)$ and $(r, \pi-\beta)$, and, together with them, the "lens shaped" domain $D(B)$ which is bounded above by the closed circular arc joining these two points, with center at the origin and radius $r>0$, and lying in the upper half plane $y>0$; and is bounded below by the straight line segment joining these two points, also lying in the half plane $y>0$, and which is a subset of the straight line of equation $y=r \sin \beta$. If Green's first identity (4) is applied to this domain $D(B)$, then, since $\phi$ is a solution of (GP) in $G$, the result is

$$
\int_{\partial D(B)} y^{p} \frac{\partial \phi}{\partial n} d s=0
$$

where $\partial D(\beta)$ denotes the boundary of $D(\beta)$. Written out explicitly, this means that

$$
r^{p} \int_{\beta}^{\pi-\beta}\left(\sin ^{p} \theta\right) \frac{\partial \phi}{\partial n}(r, \theta) r d \theta-\int_{-r \cos \beta}^{r \cos \beta}\left(r^{p} \sin ^{p} \beta\right) \frac{\partial \phi}{\partial y}(x, r \sin \beta) d x=0 .
$$

Therefore, in view of the inequality (8), 


$$
\begin{aligned}
\left|r \int_{\beta}^{\pi-\beta}\left(\sin ^{p} \theta\right) \frac{\partial \phi}{\partial n}(r, \theta) d \theta\right| & \leq \int_{-r \cos \beta}^{r \cos \beta}\left(\sin ^{p} \beta\right)\left|\frac{\partial \phi}{\partial y}(x, r \sin \beta)\right| d x \\
& \leq 4 M r\left(\sin ^{p+1} \beta\right)(r \cos \beta) /|p|,
\end{aligned}
$$

and this means that ( since $p+1>0$, and $p \neq 0$ ),

$$
\lim _{\beta \rightarrow+0} \int_{\beta}^{\pi-\beta}\left(\sin ^{p} \theta\right) \frac{\partial \phi}{\partial n}(r, \theta) d \theta=0 \text {. }
$$

(This last equation holds, in particular, for $r=\alpha R$ and for $r=R$, and will be used below for these two particular values of $r$.)

Let us now apply the identity (5) to the closure of the domain $D(\alpha, \beta)$. We take $\phi^{*}$ to be the function $\phi^{*}(r)=\left(r^{-p}\right) / p$, where $r^{2}=x^{2}+y^{2}$ and $p \neq 0$ (it is at this point of the proof that the restriction $p \neq 0$ is again essential). This function $\phi^{*}$ can easily be shown to be a solution of (GP) which is regular for $y>0$. Since all points of the closure of $D(\alpha, \beta)$ lie above the $x$ axis, and since $\phi$ is regular there, then $\operatorname{div}\left(y^{p} \operatorname{grad} \phi\right)=\operatorname{div}\left(y^{p} \operatorname{grad} \phi^{*}\right)=0$ on $D(\alpha, \beta)$, and the integral over $D(\alpha, \beta)$ vanishes. The final result is:

$$
\int_{\partial D(\alpha, \beta)} y^{p}\left(\phi^{*} \frac{\partial \phi}{\partial n}-\phi \frac{\partial \phi^{*}}{\partial n}\right) d s=0 \text {, }
$$

where $\partial D(\alpha, \beta)$ denotes the boundary of $D(\alpha, \beta)$.

Now, along any radius, $\frac{\partial \phi^{*}}{\partial n} \equiv 0 ;$ on the circular arc of radius $R$, $\frac{\partial \phi^{*}}{\partial n}=-R^{-p-1} ;$ and, on the circular arc of radius $\alpha R, \frac{\partial \phi^{*}}{\partial n}=(\alpha R)^{-p-1}$. Hence,

(11) $-\int_{\partial D(\alpha, \beta)} y^{p} \phi \frac{\partial \phi^{*}}{\partial n} d s=\int_{\beta}^{\pi-\beta} \phi(R, \theta) \sin ^{p} \theta d \theta-\int_{\beta}^{\pi-\beta} \phi(\alpha R, \theta) \sin ^{p} \theta d \theta$. Now, for $p+1>0$, the definite integral

$$
\int_{0}^{\pi} \sin ^{p} \theta d \theta
$$

is improper, but convergent, as follows from the well known inequalities 
$2 \theta / \pi \leq \sin \theta \leq \theta$, for $0 \leq \theta \leq \frac{\pi}{2}$. Consequently, making use of the uniform convergence over $0 \leq \theta \leq \pi$ in (7), the desired result (WT) would seem to follow upon first taking the limit as $\beta$ approaches 0 in (11), and then taking the limit as $\alpha$ approaches 0 , in succession. That is

$$
\lim _{\beta \rightarrow+0}\left[-\int_{\partial D(\alpha, \beta)} y^{p} \phi \frac{\partial \phi^{*}}{\partial n} d s\right]=\int_{0}^{\pi} \phi(R, \theta) \sin ^{p} \theta d \theta-\int_{0}^{\pi} \phi(\alpha R, \theta) \sin ^{p} \theta d \theta,
$$

and

(12) $\lim _{\alpha \rightarrow+0} \lim _{\beta \rightarrow+0}\left[-\int_{\partial D(\alpha, \beta)} y^{p} \phi \frac{\partial \phi^{*}}{\partial n} d s\right]=$

$$
=\int_{0}^{\pi} \phi(R, \theta) \sin ^{p} \theta d \theta-\int_{0}^{\pi} \phi_{0} \sin ^{p} \theta d \theta
$$

Consequently, in order to complete the proof of (WT), it only remains to verify that (see (10)):

$$
\lim _{\alpha \rightarrow+0} \lim _{\beta \rightarrow+0}\left[\int_{\partial D(\alpha, \beta)} y^{p_{\phi}} \frac{\partial \phi}{\partial n} d s\right]=0 .
$$

Now, when written out explicitly, the integral is

$$
\text { (14) } \begin{aligned}
\int_{\partial D(\alpha, \beta)} y^{p_{\phi^{*}} \frac{\partial \phi}{\partial n} d s=\left(\sin ^{p_{\beta}}\right) \int_{\alpha R}^{R} r^{p_{\phi^{*}}(r)\left[\phi_{n}(r, \beta)+\phi_{n}(r, \pi-\beta)\right] d r}} \\
+(\alpha R)^{p+1} \phi^{*}(\alpha R) \int_{\beta}^{\pi-\beta} \phi_{n}(\alpha R, \theta) \sin ^{p} \theta d \theta \\
+R^{p+1} \phi^{*}(R) \int_{\beta}^{\pi-\beta} \phi_{n}(R, \theta) \sin ^{p} \theta d \theta,
\end{aligned}
$$

where the definition of the auxiliary function $\phi^{*}$ is to be kept in mind. As $\beta$ approaches zero, the last two terms on the right hand side of equation (14) approach zero, by equation (9) for the particular values $r=\alpha \dot{R}$ and $r=R$. Further, from inequality (8) for $\left|\phi_{y}\right|$, and the common bound $M$ for all the first and second partial derivatives of $\phi$ in $G$, we obtain 


$$
\begin{aligned}
\left|\phi_{n}(r, \beta)\right| & =\left|\phi_{x}(r, \beta) \sin \beta-\phi_{y}(r, \beta) \cos \beta\right| \\
& \leq M \sin \beta+(2 r \sin \beta) M \cos \beta /|p| \\
& \leq(M \sin \beta)(1+2 R /|p|),
\end{aligned}
$$

and the same upper bound is valid for $\left|\phi_{n}(r, \pi-\beta)\right|$, yielding

$$
\left|\phi_{n}(r, \beta)\right|+\left|\phi_{n}(r, \pi-\beta)\right| \leq(2 M \sin \beta)(1+2 R /|p|) .
$$

Using the definition of the auxiliary function $\phi^{*}$, the inequality (16), and the inequality $p+1>0$, it follows that the first term on the right hand side of (14) also approaches zero as $\beta$ approaches 0 . Therefore, from (14) we have that

$$
\lim _{\beta \rightarrow+0} \int_{\partial D(\alpha, \beta)} y^{p} \phi^{*} \frac{\partial \phi}{\partial n} d s=0,
$$

which means that

$$
\lim _{\alpha \rightarrow+0} \lim _{\beta \rightarrow+0} \int_{\partial D(\alpha, \beta)} y^{p_{\phi}} \frac{\partial \phi}{\partial n} d s=0
$$

The desired result, (WT), now follows directly from (10), (12) and (18), and the proof is complete.

It is a corollary that the theorem holds for all $p>-1$, provided we require, for $p=0$, that $\phi_{y}(x, 0) \equiv 0$ on $I$. In fact, when $p=0$, the harmonic function $\phi$ can then be continued into the lower half-plane as an even function of $y$, and (WT) is equivalent to the classical Gauss mean value theorem for harmonic functions.

3. Weinstein's mean value theorem for $p \leq-1$ ?

It is clear from (WT), however, that the theorem cannot hold for $p \leq-1$, unless (possibly) restrictions are placed on $\phi$. The same conclusion can be reached, in a quite intuitive formal manner, by recalling Weinstein's concept of $\phi$ as an axially symmetric potential in a fictitious space of $(p+2)$ dimensions (for definiteness, if desired, think of $p$ as a positive integer). Indeed, if $d S$ and $r$ are, respectively, the surface element and radius of a sphere, centered at the origin, in $(p+2)$-dimensional space, and, if $\omega_{p+1}$ is the surface area of a $(p+1)-$ dimensional unit sphere, then the classical Gauss mean value relation for 
the sphere (for harmonic functions), becomes, by virtue of the axial symetry, a relation on a meridian plane:

$$
\begin{aligned}
\phi_{0} & =\frac{\int \phi d S}{\int 1 d S}=\frac{\int_{0}^{\pi} \phi y^{p} \omega_{p+1} d \theta}{\int_{0^{2}}^{\pi} \omega_{p+1} d \theta} \\
& =\frac{\omega_{p+1} \int_{0}^{\pi} \phi(r \sin \theta)^{p} d \theta}{\omega_{p+1} \int_{0}^{\pi}(r \sin \theta)^{p} d \theta}=\frac{\int_{0}^{\pi} \phi \sin p_{\theta d \theta}}{\int_{0}^{\pi} \sin ^{p} \theta d \theta} .
\end{aligned}
$$

This is precisely (WT), and it is apparent that the Weinstein Theorem concerns the mean value of a symmetric harmonic function on a sphere in $(p+2)$-dimensional space. Now, one of these $(p+2)$ dimensions must be assigned to the axis of symmetry, leaving $(p+1)$ "residual" dimensions at our disposal for constructing the $(p+2)$-dimensional sphere about the axis of symmetry. Unless $p>-1$, however, no "residual" dimensions are available for constructing the sphere, and this intuitive interpretation of the theorem fails. Such considerations must, of course, be purely formal, without a definition of nonintegral dimensionality.

Can "analytic continuation" (with respect to the real variable $p$, starting with the interval $p>-1$, and then proceeding to the interval $p \leq-1$; compare, for example, Riesz [3], Diaz and Ludford [1], Diaz and Weinberger [2]); or, alternatively, Weinstein's "correspondence principle" (compare, for example, Weinstein [5]), be employed to provide an answer to the question formulated in the title of this section?

\section{References}

[1] J.B. Diaz and G.S.S. Ludford, "On the Euler-Poisson-Darboux equation, integral operators, and the method of descent", Proc. Conf. Diff. Equ., University of Maryland, March 1955, 73-89 (University of Maryland Book Store, College Park, Maryland; 1956). 
[2] J.B. Diaz and H.F. Weinberger, "A solution of the singular initial value problem for the Eller-Poisson-Darboux equation", Proc. Amer. Math. Soc. 4 (1953), 703-715.

[3] Marcel Riesz, "L'intégrale de Riemann-Liouville et le problème de Cauchy", Acta Math. 81 (1949), 1-223.

[4] Alexander Weinstein, "Discontinuous integrals and generalized potential theory", Trans. Amer. Math. Soc. 63 (1948), 342-354.

[5] Alexander Weinstein, "Generalized axially symmetric potential theory", Buzz. Amer. Math. Soc. 59 (1953), 20-38.

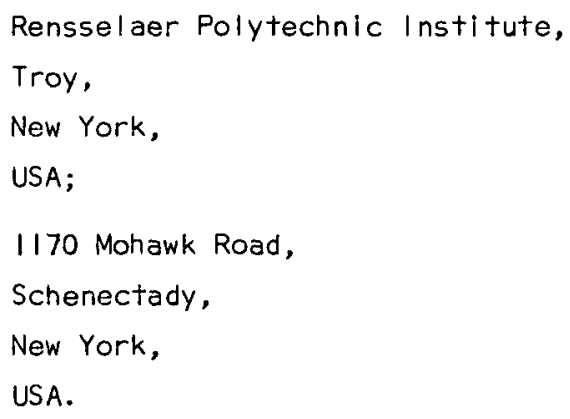

